\title{
Variables determining clinical complexity in hospitalized Internal Medicine patients: a workload analysis
}

\author{
Valentina Tommasi, ${ }^{1,2}$ Alessandra Campolongo, ${ }^{2}$ Irene Caridi, ${ }^{1}$ Simone Gatti, ${ }^{1}$ Lorena Lagana, ${ }^{2}$ Ilaria Simonelli, ${ }^{2}$ \\ Paola Piccolo, ${ }^{1}$ Dario Manfellotto ${ }^{1,2}$ \\ ${ }^{1}$ Internal Medicine Unit, S. Giovanni Calibita, Fatebenefratelli Hospital; ${ }^{2}$ Fatebenefratelli Research Foundation for Healthcare \\ and Social Education, Fatebenefratelli Hospital, Isola Tiberina, Roma, Italy
}

\begin{abstract}
The clinical complexity of Internal Medicine patients is a daily challenge for clinicians. Although clinical complexity cannot be directly measured, several scores describe the variability of clinical severity and comorbidity. The aim of this study was to analyze staff workload by assessing the nursing and medical complexity of patients admitted to an Internal Medicine ward. We included 40 consecutive inpatients [ $52.5 \%$ females, mean age 71.2 (18.2) years] classified according to the index of clinical complexity (ICC, type A: very high; type B: high; type C: moderate) and the cumulative illness rating scale (CIRS) severity and comorbidity index. Patient outcomes, hospitalization duration, tests performed, number of daily medications and time to perform standard nursing tasks were analysed across groups. Mean duration of hospitalization was 15.6 (10.1) days; in-hospital mortality was $15 \%$. Mean CIRS severity index (SI) was 1.03 (0.31) and median CIRS comorbidity index (CI) was 2 (range 1-5). Significant differences were observed among ICC groups in time spent performing specific tasks [univariate analysis of variance $\mathrm{F}(2.37)=17.26, \mathrm{P}<0.001]$. No significant differences were found between the three groups for mean CIRS-SI [F(2.37)=3.033, $\mathrm{P}=0.060$ ] and median CIRS-CI [Kruskal Wallis test: $\mathrm{c}^{2}(2)=1.672, \mathrm{P}=0.433$ ]. Clinical complexity and caring complexity were not correlated in our sample of Internal Medicine inpatients. Optimal care of Internal Medicine patients must take into account their complexity in both the medical and nursing aspects.
\end{abstract}

\section{Introduction}

The elevated costs of healthcare require continuous development to obtain greater efficacy of activities, and to satisfy a constantly growing demand, despite the lack of resources. ${ }^{1,2}$ The hospital is a very

Correspondence: Valentina Tommasi, Internal Medicine Unit, S. Giovanni Calibita, Fatebenefratelli Hospital, Isola Tiberina, piazza Fatebenefratelli 1, 00186 Roma, Italy.

Tel.: +39.06.6837260. E-mail: vxilit@tiscali.it

Key words: Index of caring complexity; cumulative illness rating scale; nursing workload.

Award: Premio Ricerca FADOI Lazio 2015 - $3^{\text {rd }}$ place.

Conflict of interest: the authors declare no potential conflict of interest.

Received for publication: 8 April 2016

Revision received: 12 October 2016.

Accepted for publication: 17 October 2016.

This work is licensed under a Creative Commons Attribution NonCommercial 4.0 License (CC BY-NC 4.0).

CCopyright V. Tommasi et al., 2017

Licensee PAGEPress, Italy

Italian Journal of Medicine 2017; 11:202-206

doi:10.4081/itjm.2017.725 complex system that is influenced by a great number of interconnected variables and needs a representative model that can objectively define and describe the different components, testing the efficacy of strategic choices to provide mechanisms for decision makers in health services planning and management. Nowadays hospitals must respect the principles of cost containment and improvement of healthcare quality standards. Therefore, the objective analysis of clinical activities can highlight the areas in which problems can arise, allowing the healthcare system management to make corrections and fully take advantage of available resources. ${ }^{3}$

One of the most important problems of healthcare systems is the objective evaluation of the staff workload. Without an instrument to measure workload, it is very difficult to demonstrate the amount of work performed by the staff, and the management's decisions regarding staff hiring and allocation to different wards may not reflect actual requirements, leading to lower standard of care., ${ }^{4,5}$

In the absence of a specific tool to measure daily workload, for example, in most cases nursing staff is assigned to hospital wards based solely on the number of beds. ${ }^{6}$

In the setting of Internal Medicine wards, clinical complexity of patients and the varying intensity levels of required care clearly suggests the need for tools to measure activity. ${ }^{7}$ Monitoring the workloads of clini- 
cians and nurses allows a better resource allocation, improvement in clinical services and increasing economic and clinical effectiveness. Assessing the most frequent staff activities allows to better distribute activities among professionals, identify staffs' competencies and activities to be prescribed to health-care support workers. ${ }^{8-10}$

Among different methods of nursing classification, the index of caring complexity (ICC) is a management tool to evaluate appropriateness of nursing procedures, integrate health-care support workers and to monitor the need of nursing staff. Methodology does not require specific data collection but the classification derives directly from the data flow obtained during the caring process as a health/disease continuum..$^{10,11}$

On the other hand, clinical complexity takes into account different variables, such as number of comorbid conditions, number of daily medications, need for repeated hospitalizations over a given time period. ${ }^{12}$

Comorbidity is defined as the coexistence of two or more disease conditions in the same subject, and is typical of the elderly; with advancing age, an increase in age-related chronic degenerative diseases is expected. ${ }^{13}$

Comorbidities increase the duration of hospitalization, the need for re-hospitalizations, the incidence of complications and the risk of mortality compared to that of the single disease components. ${ }^{14}$

The cumulative illness rating scale (CIRS) was originally designed by Linn et al., to specifically measure comorbidities among hospital inpatients. The CIRS classifies comorbidities by affected organs/systems, and defines for each category a severity grading from 0 to 4: where 0 indicates the absence of the disease, $1=$ mild, $2=$ moderate, $3=$ severe and $4=$ very severe. ${ }^{15,16}$

All these variables are intuitively related to workload in terms of minutes of nurses' care giving, however there is no objective evaluation method to correlate workload to the patients' clinical complexity.

The aim of this pilot study was to prospectively analyze the variables determining caring and clinical complexity of patients admitted to an Internal Medicine ward classified according to the CIRS and ICC scales, and to assess the reliable and objectively quantifiable method to evaluate nurses' workload for optimal human resources allocation.

\section{Materials and Methods}

This prospective pilot study assessed healthcare staff activities in the Internal Medicine Unit of a secondary urban Hospital in Rome, Italy. From November 1 to November 30, 2014, consecutive patients admitted for more than $48 \mathrm{~h}$ were enrolled.

Healthcare providers provided informed consent to participate in the study while performing their standard clinical duties according to good clinical practice, and patients consented to data collection from medical records.

Demographic data, number of patients' diagnoses, number of clinical/radiological test performed, number of prescribed daily medications, hospitalization duration, and outcome were collected from patient charts.

Medical complexity was measured by assigning CIRS scores for severity and comorbid conditions. Briefly, 14 categories of disease are considered (cardiac, hypertensive, vascular, respiratory, ocular, ear-nosethroat, upper gastrointestinal, lower gastrointestinal, hepatic, renal, genitourinary, musculoskeletal/cutaneous, nervous, endocrine-metabolic, psychiatric-behavioral). The CIRS classifies comorbidities by affected organs/ systems, and defines for each category a severity grading from 0 to 4 : where 0 indicates the absence of the disease, $1=$ mild, $2=$ moderate, $3=$ severe and $4=$ very severe.

Two scores are obtained: the severity index (SI), from the mean score of the first 13 categories (score range $0-4)$; comorbidity index (CI), the number of categories (among the first 13) with an $\mathrm{SI} \geq 3$ (maximum score $=13)$.

The extent of caring complexity was assessed by identifying standard caring activities (e.g., registration for admission, preparing and placing the patient in bed, vital signs assessment and recording, patient preparation for blood tests and electrocardiogram; Table 1). Each staff member subjectively assessed predicted time needed to perform each activity; duration of each task was then objectively assessed in minutes with a chronometer. For each activity, we identified the timing of execution (daily activity taking place during the hospitalization or at discharge); frequency of the activity; duration of execution in minutes.

Patients were classified considering the $\mathrm{ICC}^{10}$ into 3 groups of decreasing caring complexity: type A: very high complexity; type B: high complexity; type $\mathrm{C}$ : moderate complexity.

\section{Statistical analysis}

Data are presented as mean (standard deviation, $\mathrm{SD}$ ) or median (range) if not normally distributed. Clinical variables, CIRS score, admission outcomes were compared among ICC groups with chi-square test (or Fisher exact test), parametric [univariate analysis of variance (ANOVA)] or a non-parametric test (Kruskal-Wallis test) as appropriate. Correlation was evaluated performing a parametric (Pearson's correlation coefficient) or, when appropriate, a nonparametric (Spearman's rank correlation coefficient) correlation coefficient calculation. Multiple comparisons were performed and Bonferroni's correction was applied. A P-value less than 0.05 was considered significant. Data analyses were performed with SPSS 16.0 (IBM Corp., Armonk, NY, USA). 


\section{Results}

During the study period, 40 consecutive inpatients were enrolled $(52.5 \%$ females, mean age 71.2 $(\mathrm{SD}=18.2)$ years, median age 78 years, range 23-92). Mean duration of hospitalization was $15.6(\mathrm{SD}=10.1)$ days (median 13 days, range 2-46). Overall 6 patients died in-hospital $(15 \%), 5$ were transferred to other wards $(12.5 \%)$ and 29 were discharged $(72.5 \%)$. Overall, mean CIRS-SI was $1.03(\mathrm{SD}=0.31)$ and median CIRS-CI was 2 (range 1-5) equivalent to a moderate comorbidity. The mean number of diagnoses at discharge was $5.9(\mathrm{SD}=2.3)$, the median number of clinical tests performed during hospitalization was 8 (range 3-18), and the mean number of medications prescribed at discharge was $8(\mathrm{SD}=3.2)$.

Based on the ICC scale, 22 patients were type A (55\%), 10 patients were type B (25\%), and 8 patients were type $\mathrm{C}(20 \%)$. No significant association with gender was observed $\left[\mathrm{c}^{2}(2)=0.863, \mathrm{P}=0.684\right]$. Comparisons among ICC patients' groups in baseline demographic and hospitalization parameter data were showed in Table 2.

Significant differences were observed among ICC groups in time spent performing specific nursing tasks [univariate $\operatorname{ANOVA} F(2.37)=17.26, \mathrm{P}<0.001]$. By the multiple comparison a significant difference was found between the time spent by healthcare assistants performing hygienic care for type A patients compared to type $\mathrm{B}$ or for type $\mathrm{C}(\mathrm{P}=0.015$ and $\mathrm{P}<0.001$, respectively). The difference in time was also significant between type $\mathrm{B} v s$ type $\mathrm{C}$ patients $(\mathrm{P}=0.042)$. Time spent performing blood sampling was different between patients' types [Kruskal Wallis test: $\mathrm{c}^{2}(2)=20.17$, $\mathrm{P}<0.001$ ]; the time of performing blood sampling in type A patients was greater than that of type $\mathrm{B}$ $(\mathrm{P}<0.001)$ or than type $\mathrm{C}$ patients $(\mathrm{P}<0.001$, Table 3$)$.

No significant differences were found between the three groups of patients in the mean CIRS-SI $(\mathrm{F}(2.37)=3.033, \mathrm{P}=0.060)$ and in median CIRS-CI score [Kruskal Wallis test: $\left.\mathrm{c}^{2}(2)=1.672, \mathrm{P}=0.433\right]$. No statistically significant correlation was found between CIRS severity and comorbidity indices and each of the examined caring activities (all $\mathrm{P}>0.087$ ).

\section{Discussion}

Internal Medicine patients are by nature complex due to advanced age, comorbid chronic conditions, numerous concomitant pharmacological treatments with a high potential for drug-drug interactions. This clinical complexity translates into greater workload for healthcare providers, especially in the hospital setting. ${ }^{12}$

To the best of our knowledge, an objective measure of patient's clinical complexity, considering all the characteristics and variables of a specific health care setting, is lacking. ${ }^{17,18}$

The major weakness of the used methods is that

Table 1. Comprehensive list of clinical staff activities considered for objective assessment.

\begin{tabular}{ll}
\hline Description of activities & When the activity is performed \\
\hline Registration for admission & First day of hospitalization \\
\hline Preparing and placing the patient in bed & First day of hospitalization \\
\hline Patient preparation for blood tests and electrocardiogram & First day of hospitalization \\
\hline Patient preparation for image testing & First day of hospitalization \\
\hline Patient preparation for oxygen therapy & First day of hospitalization \\
\hline Hygienic care & 3 times a day \\
\hline Therapy administration & 3 times a day \\
\hline Positioning of venous access & When needed \\
\hline Diagnostic tests & At admission and when needed \\
\hline Vital signs & 3 times a day \\
\hline Urinary catheterization & Every 15 days \\
\hline Urine bag replacement and diuresis monitoring & 2 times a day \\
\hline Dressings & When needed \\
\hline Diaper change & 3 times a day \\
\hline Counseling family members on discharge recommendations & At hospital discharge \\
\hline Staff shift change briefing & 3 times a day \\
\hline Patient medical chart compilation and print & After the hospital discharge \\
\hline
\end{tabular}


the staff workload is conceptualized at a macro level, without taking into account the specific organizational characteristics (e.g., physical layout, information technology available) that may significantly affect workload. ${ }^{19}$ When performing their tasks, clinicians and nurses encounter different situations and patients, which are determinants of the situation- and patientlevel workloads. The lack of objectivity in estimation of length activity reinforces the necessity of an instrument that permits a precise measure of staff workloads. These instruments play a fundamental role in terms of organization, allowing improving performance, to reduce costs, to reduce critical situations.

A specific quantitative analysis of activities allows identifying elements to be corrected or improved, such as time lost, new activities/learning curves, and the clinical and organizational consequences of excessive staff workloads.

In the present study, we describe severity of complex Internal Medicine patients based on hospitalization parameters and CIRS score, as well as complexity of care based on specific nursing activities. As expected, an association between activity execution time and patient complexity was observed, with the more care complex patients requiring longer duration of activity execution. Demographic data confirm that the less complex ICC group (group C) consisted of the youngest patients with the lowest number of prescribed medications; however, the duration of hospitalization and the number of tests prescribed during admission did not differ from higher complexity class patients (groups A and B). The oral interview (data not shown) of predicted duration of caring activities showed that perceived time to perform specific tasks is subjective and depends on the single healthcare worker. As expected, objective measurement of execution time confirmed that non self-sufficient patients present in the ICC group A require more assistance for activities such as hygienic care and blood sampling; however, no differences were found compared to less complex patients in the execution of the other standard caring activities. Although the ICC does not show a

Table 2. Comparisons of clinical and hospitalization outcome parameters among index of clinical complexity groups.

\begin{tabular}{llcccc}
\hline Parameters & & $\begin{array}{c}\text { Type } \mathbf{A} \\
\mathbf{n = 2 2}\end{array}$ & $\begin{array}{c}\text { Type B } \\
\mathbf{n = 1 0}\end{array}$ & $\begin{array}{c}\text { Type C } \\
\mathbf{n = 8}\end{array}$ & $\mathbf{P}$ \\
\hline Male gender & $\mathrm{N}(\%)$ & $8(36.4 \%)$ & $6(60 \%)$ & $5(62.5 \%)$ & $0.332^{*}$ \\
\hline Mean age (years) & Mean (SD) & $80(9.76)$ & $76.2(9.72)$ & $40.7(10.22)$ & $<0.001^{* *}$ \\
\hline Mortality & $\mathrm{N}(\%)$ & $6(27.3 \%)$ & 0 & 0 & $0.075^{*}$ \\
\hline Hospitalization (days) & Mean (SD) & $19.4(12.54)$ & $10.4(4.25)$ & $12(6.70)$ & $0.049^{* *}$ \\
\hline Number of diagnoses & Mean (SD) & $6.5(2.40)$ & $5.8(1.55)$ & $4(1.77)$ & $0.023^{* *}$ \\
\hline Number of tests performed & Mean (SD) & $7.8(3.67)$ & $8.7(2.50)$ & $7(3.21)$ & $0.557^{* *}$ \\
\hline Number of drugs prescribed & Mean (SD) & $8.7(3.18)$ & $9.1(2.33)$ & $4.8(2.61)$ & $0.004^{* *}$ \\
\hline CIRS-SI & Mean (SD) & $1.1(0.3)$ & $1.1(0.3)$ & $0.8(0.2)$ & $0.060^{* *}$ \\
\hline CIRS-CI & Median (min-max) & $2(1-5)$ & $2.5(1-5)$ & $2(1-4)$ & $0.433^{* * *}$
\end{tabular}

Type A, very high complexity; Type B, high complexity; Type C, moderate complexity; SD, standard deviation; CIRS, cumulative illness rating scale; SI, severity index; CI, comorbidity index. *P-values referred to Fisher exact test; **P-values referred to univariate analysis of variance; ***P value referred to Kruskal Wallis test.

Table 3. Comparisons of time required (in minutes) by nursing staff to perform clinical tasks between index of clinical complexity groups.

\begin{tabular}{llcccc}
\hline Nursing activities & & $\begin{array}{c}\text { Type } \mathbf{A} \\
\mathbf{n = 2 2}\end{array}$ & $\begin{array}{c}\text { Type B } \\
\mathbf{n = 1 0}\end{array}$ & $\begin{array}{c}\text { Type C } \\
\mathbf{n = 8}\end{array}$ & P* \\
\hline Hygienic care & Mean (SD) & $12.1(4.7)$ & $8(1.25)$ & $3.6(0.9)$ & $<0.001$ \\
\hline Blood sampling & Median (min-max) & $4(3-6)$ & $2(2-4)$ & $2.5(2-3)$ & $<0.001$ \\
\hline Changing bed linen & Median (min-max) & $5(2-8)$ & $3(2-5)$ & $4(2-5)$ & 0.078 \\
\hline Therapy administration & Median (min-max) & $5(2-15)$ & $5(3-11)$ & $4(2-8)$ & 0.419 \\
\hline Examination of BP, T, HGT parameters & Median (min-max) & $4(2-5)$ & $3(2-5)$ & $3.5(3-5)$ & 0.111 \\
\hline Patient data registration & Median (min-max) & $1(0-4)$ & $2(1-2)$ & $2(1-2)$ & 0.380 \\
\hline
\end{tabular}

Type A, very high complexity; Type B, high complexity; Type C, moderate complexity; SD, standard deviation; BP, blood pressure; T, body temperature; HGT, hemoglucotest. *Pvalues referred to univariate analysis of variance or Kruskal Wallis test. 
direct correspondence with clinical complexity, our results show that a reduction in time spent in specific caring activities is associated with lower ICC class.

Furthermore, the patients' medical complexity expressed as CIRS severity and comorbidity indices, number of tests performed during hospitalization and admission duration were not correlated with ICC class.

In accordance with previous observations, no single index can accurately characterize the complexity of Internal Medicine patients. ${ }^{18}$

\section{Limitations of the study}

A limitation of the present study is the small sample size of patients and nurses enrolled, and the lack of a multicentric evaluation of the workload on the same patients' complexity. Given these very preliminary results, we could not translate the data into a standardized application for human resources allocation related to the complexity score.

\section{Conclusions}

Nonetheless, the use of indices such as CIRS and ICC can be useful in the allocation of the human resources to an Internal Medicine ward, by providing an estimate of expected workload.

Larger prospective studies are warranted to identify reliable tools to measure workload and correctly allocate healthcare resources in Internal Medicine hospital settings.

\section{References}

1. Nardi R, Berti F, Fabbri LM, et al. Toward a sustainable and wise healthcare approach: potential contributions from hospital Internal Medicine Departments to reducing inappropriate medical spending. Ital $\mathrm{J}$ Med 2013;7:65-81.

2. Nardi R, Gardellini A, Iori I. Internal Medicine wards overcrowding and clinical risk management: structural or systemic interventions needed? Ital J Med 2009;3:3-8.

3. Cavaliere B. Sviluppo e applicazione del metodo ICA. Aspetti correlati al "carico di lavoro" e alla determinazione del fabbisogno di personale sanitario e di supporto. Sanità Pubblica Privata 2012;1:20-31.
4. Grafen M, Mackenzie FC. Development and early application of the Scottish Community Nursing Workload Measurement Tool. Br J Commun Nurs 2015;20:89-92.

5. Lobo VM, Fisher A, Ploeg J, et al. A concept analysis of nursing overtime. J Adv Nurs 2013;69:2401-12.

6. Cavaliere B, Snaidero D. Metodologia per la rilevazione della complessità assistenziale: calcolo dell'indice di Complessità Assistenziale. Manage Inferm 1999;1:32-6.

7. Nardi R, Berti F, Greco A. Complexity in hospital internal medicine departments: what are we talking about? Ital J Med Ital J Med 2013; 7:142-55.

8. Nardi R, Fabbri T, Belmonte G. Internal medicine, complexity, evidence based medicine, almost without evidences. Ital J Med 2009;3:191-200.

9. Alghamdi MG. Nursing workload: a concept analysis. J Nurs Manag 2016 [Epub ahead of print].

10. Guarinoni M, Petrucci C, Lancia L, Motta PC. The concept of care complexity: a qualitative study. Public Health Res 2015;4:588.

11. Simonetti V, Comparcini D, Buccolini M, Cicolini G. Index of caring complexity as a management tool in a surgical setting: an observational study. Prof Inferm 2014;67:235-42.

12. Nardi R, Scanelli G, Borioni D, et al. The assessment of complexity in internal medicine patients. The FADOI Medicomplex Study. Eur J Intern Med 2007;18:283-7.

13. Volpato S, Onder G, Cavalieri M, et al. Characteristics of nondisabled older patients developing new disability associated with medical illnesses and hospitalization. J Gen Intern Med 2007;22:668-74.

14. Franchi C, Nobili A, Mari D. Risk factos for hospital readmission of elderly patients. Eur J Intern Med 2013;24:45-51.

15. Linn BS, Linn MW, Gurel L. Cumulative illness rating scale. J Am Geriatr Soc 1968;16:622-6.

16. Conwell Y, Forbes NT, Cox C, Caine ED. Validation of a measure of physical illness burden at autopsy: the cumulative illness rating scale. J Am Geriatr Soc 1993; 41:38-41.

17. Kirkhus L, Jordhoy M, Saltyte Benth J, et al. Comparing comorbidity scales: attending physician score versus the cumulative illness rating scale for geriatrics. J Geriatr Oncol 2016;7:90-8.

18. Zullig LL, Whitson HE, Hastings SN, et al. A Systematic review of conceptual frameworks of medical complexity and new model development. J Gen Intern Med 2016;31:329-37.

19. Lobo E, Ventura T, Navio M, et al. Identification of components of health complexity on internal medicine units by means of the INTERMED method. Int J Clin Pract 2015;69:1377-86. 